\title{
Separation of the individual contributions to the spin Hall effect in dilute alloys within the first-principles Kubo-Středa approach
}

\author{
Kristina Chadova, ${ }^{1,}{ }^{*}$ Dmitry V. Fedorov, ${ }^{2,3}$ Christian Herschbach, ${ }^{2,3}$ Martin Gradhand, ${ }^{4}$ Ingrid Mertig, ${ }^{2,3}$ \\ Diemo Ködderitzsch, ${ }^{1}$ and Hubert Ebert ${ }^{1}$ \\ ${ }^{1}$ Department of Chemistry, Physical Chemistry, Ludwig-Maximilians University, 81377 Munich, Germany \\ ${ }^{2}$ Max Planck Institute of Microstructure Physics, Weinberg 2, 06120 Halle, Germany \\ ${ }^{3}$ Institute of Physics, Martin Luther University Halle-Wittenberg, 06099 Halle, Germany \\ ${ }^{4}$ H. H. Wills Physics Laboratory, University of Bristol, Bristol BS8 1TL, United Kingdom
}

(Received 29 April 2015; published 24 July 2015)

\begin{abstract}
We present a procedure for the separation of the intrinsic, side-jump, and skew-scattering contributions to the spin Hall conductivity within the $a b$ initio Kubo-Středa approach. Furthermore, two distinct contributions to the side-jump mechanism, either independent of the vertex corrections or solely caused by them, are quantified as well. This allows for a detailed analysis of individual microscopic contributions to the spin Hall effect. The efficiency of the proposed method is demonstrated by a first-principles study of dilute metallic alloys based on $\mathrm{Cu}, \mathrm{Au}$, and Pt hosts.
\end{abstract}

DOI: 10.1103/PhysRevB.92.045120

PACS number(s): 71.15.Rf, 72.25.Ba, 75.76.+j, 85.75.-d

\section{INTRODUCTION}

A detailed understanding of the spin Hall effect (SHE) [1] is the key issue for its efficient application in spintronic devices. The phenomenon, being connected to the anomalous Hall effect (AHE) [2-4], is a powerful tool for the creation of spin currents in nonmagnetic materials. Three main mechanisms contributing to the AHE as well as the SHE were established [5-7], all caused by spin-orbit coupling (SOC). They are known as the intrinsic mechanism due to the anomalous velocity [2,8], the extrinsic skew-scattering [3] and side-jump [4] mechanisms. In dilute alloys the skew-scattering contribution to the spin Hall conductivity dominates [9-12] since it is inversely proportional to the impurity concentration, while the other two contributions are concentration independent. Moreover, the skew-scattering mechanism is solely caused by the vertex corrections $[5,10,12,13]$. These features provide the basis for a simple separation of the skew-scattering contribution [10]. On the other hand, the intrinsic mechanism is caused exclusively by the host band structure [2], which makes it accessible by considering the corresponding ideal crystal [14-16]. The side-jump mechanism is much more subtle. Although it is caused by the presence of impurities in a host, the corresponding contribution to the spin Hall conductivity does not depend on their concentration [5]. Furthermore, for uncorrelated short-range disorder it is even independent of the type of impurities [17]. In contrast to the skew scattering, the side-jump mechanism is not only caused by the vertex corrections but has a contribution independent of them [5]. This complicates its coherent description, and quite often different approximations are used. For instance, in Refs. [12,18] the influence of the vertex corrections was neglected for the semiclassical resonant scattering model proposed to describe the side-jump contribution to the spin Hall conductivity. On the other hand, in Ref. [10] only the part caused by the vertex corrections was considered, which together with the skew-scattering contribution can be elegantly separated

\footnotetext{
*kchpc@cup.uni-muenchen.de
}

from the rest [19]. However, for a complete description and comparison of the different mechanisms contributing to the SHE, it is highly desirable to have a consistent and fully $a b$ initio treatment of the side-jump contribution.

\section{APPROACH AND RESULTS}

In this paper we propose an efficient procedure for the separation of the three main contributions, as well as the two parts related to the side-jump mechanism, schematically illustrated by Fig. 1. Practically, this is realized by means of the firstprinciples Kubo-Streda approach as implemented within the multiple-scattering Korringa-Kohn-Rostoker Green-function method [10]. Computational details of the method used can be found in the Appendix. Our procedure is based on the commonly accepted decomposition of the total spin Hall conductivity (SHC),

$$
\sigma_{x y}^{z}=\sigma_{x y}^{z \text { intr }}+\sigma_{x y}^{z \text { sj }}+\sigma_{x y}^{z \text { skew }}
$$

into its intrinsic (intr), side-jump (sj), and skew-scattering (skew) contributions [5-7]. In addition, for the side-jump contribution we perform the decomposition

$$
\sigma_{x y}^{z \mathrm{sj}}=\sigma_{x y}^{z \mathrm{sj}(\mathrm{nvc})}+\sigma_{x y}^{z \mathrm{sj}(\mathrm{vc})}
$$

where the first and second terms represent the parts independent of the vertex corrections and solely caused by them,

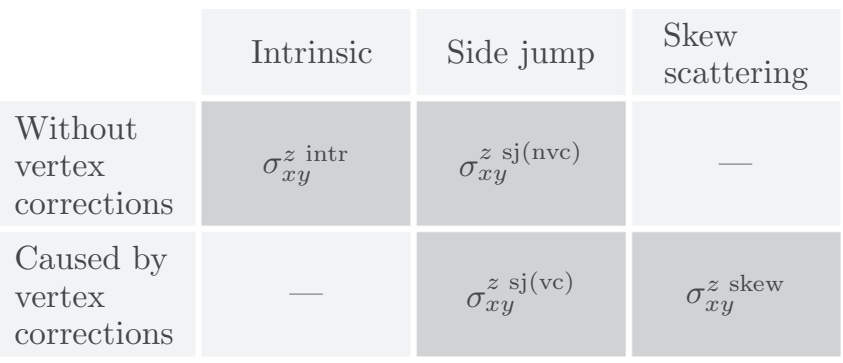

FIG. 1. Schematic representation of the considered individual contributions to the spin Hall effect. 
respectively. Within the present work, we quantify the four distinct contributions from Eqs. (1) and (2) by applying the proposed separation technique to $\mathrm{Cu}, \mathrm{Au}$, and $\mathrm{Pt}$ hosts with $5 d$ impurities.

The so-called anomalous-distribution and intrinsic skewscattering contributions, which were additionally singled out in Ref. [5], are assumed to be included in $\sigma_{x y}^{z \mathrm{sj}(\mathrm{nvc})}$ and $\sigma_{x y}^{z \mathrm{sj}(\mathrm{vc})}$, respectively. For the former one this is quite natural since the anomalous distribution is caused by the longitudinal component of the coordinate shift [20], whose transversal component to an applied electric field is responsible for the side-jump velocity [5]. Moreover, the anomalous-distribution contribution arises without the vertex corrections. By contrast, the intrinsic skew-scattering contribution arises due to the vertex corrections as well as the asymmetric scattering rate [21], similar to its conventional counterpart represented in our analysis by $\sigma_{x y}^{z \text { skew }}$. However, in comparison to this quantity, the intrinsic skew-scattering contribution is independent of the impurity concentration [5,21], which makes it similar to the side-jump mechanism. Therefore, here we follow Ref. [7] in parsing the mechanisms, where this contribution was formally attributed to the side-jump scattering.

In the dilute limit, $\sigma_{x y}^{z s k e w}$ is inversely proportional to the impurity concentration, which also holds for the longitudinal charge conductivity $\sigma_{x x}$. This allows one to rewrite Eq. (1) as [10]

$$
\sigma_{x y}^{z}\left(\sigma_{x x}\right)=\sigma_{x y}^{z \text { intr }}+\sigma_{x y}^{z \mathrm{sj}}+\alpha^{\text {skew }} \sigma_{x x},
$$

where $\alpha^{\text {skew }}=\sigma_{x y}^{z \text { skew }} / \sigma_{x x}$ is the spin Hall angle related to the skew scattering. Then, the separation of the sum of the intrinsic and side-jump contributions from the skew-scattering contribution can be done by extrapolating to the situation of vanishing charge conductivity

$$
\sigma_{x y}^{z \text { intr }}+\sigma_{x y}^{z \mathrm{sj}}=\sigma_{x y}^{z}\left(\sigma_{x x} \rightarrow 0\right) .
$$

At low impurity concentrations $\sigma_{x y}^{z}$ shows a linear behavior as a function of $\sigma_{x x}$ [10], which is discussed in more detail in the Appendix. Such a typical situation is shown in Fig. 2 by the results obtained for dilute $\mathrm{Cu}(\mathrm{Au})$ alloys [22].

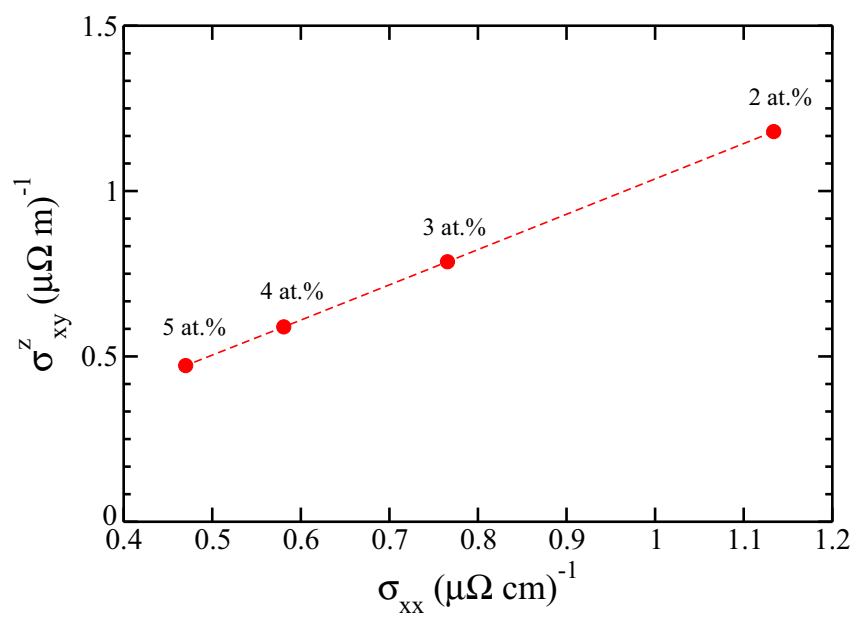

FIG. 2. (Color online) The spin Hall conductivity as a function of the longitudinal charge conductivity is shown for four dilute $\mathrm{Cu}(\mathrm{Au})$ alloys labeled by the corresponding impurity concentrations.
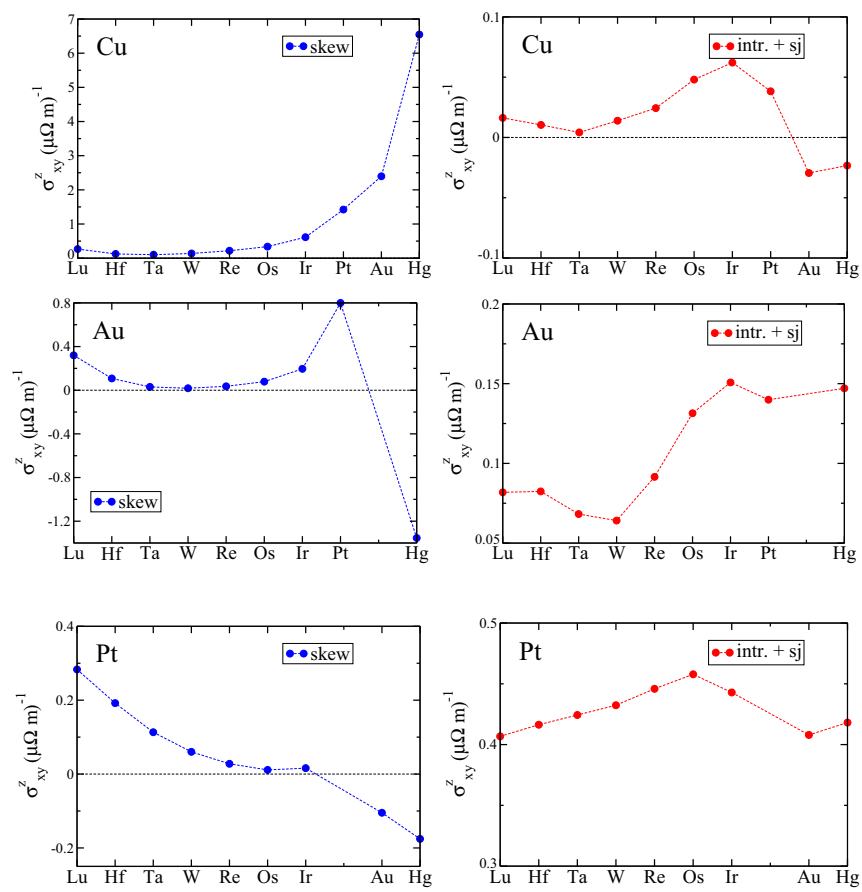

FIG. 3. (Color online) The skew-scattering contribution $\sigma_{x y}^{\text {zskew }}$ (left) at the impurity concentration 1 at. $\%$ as well as the sum of the intrinsic and side-jump contributions $\sigma_{x y}^{z \text { intr }}+\sigma_{x y}^{z \text { sj }}$ (right) are shown as obtained for different dilute alloys based on $\mathrm{Cu}, \mathrm{Au}$, and Pt hosts.

Due to the linear behavior of the SHC as a function of the charge conductivity, it is possible to perform an extrapolation using just a few points. This gives us the sum $\sigma_{x y}^{z \text { intr }}+\sigma_{x y}^{z s j}$, whose subtraction from the total SHC provides the skewscattering contribution as well. The corresponding results for $\mathrm{Cu}, \mathrm{Au}$, and $\mathrm{Pt}$ hosts with different $5 d$ impurities are shown in Fig. 3 (left panels). Going from $\mathrm{Cu}$ to $\mathrm{Pt}$ via $\mathrm{Au}$, the skew-scattering contribution decreases since the difference between impurity and host SOC becomes smaller [13]. On the other hand, the increase of the host SOC enhances the sum shown in the right panels of Fig. 3. In order to demonstrate that this effect is caused by the enhancement of the intrinsic contribution, we need to perform a further separation of the individual contributions $\sigma_{x y}^{z \text { intr }}$ and $\sigma_{x y}^{z \mathrm{sj}}$.

A possible way to access the intrinsic contribution to the SHC is based on the Berry curvature formalism [15,23]. To obtain this contribution within the used Kubo-Středa approach, we employ a scheme similar to the one proposed and successfully applied to pure metals in case of the AHE [24]. It implies an addition of a small imaginary part $i \epsilon$ to the Fermi energy $\left(E_{F}\right)$, in order to calculate $\sigma_{x y}^{z}$ given by Eq. (3) of Ref. [10] via an integration over the Brillouin zone (BZ). Due to this procedure, we avoid the numerical problems caused by a $\delta$-function-like behavior of the integrand at the real energy axis, which is present for pure crystals [24]. Then, the intrinsic contribution to the SHC can be obtained by the extrapolation $\lim _{\epsilon \rightarrow 0} \sigma_{x y}^{z}\left(E_{F}+i \epsilon\right)$. Applying this scheme with the parameters described in the Appendix, we have obtained $\sigma_{x y}^{z \text { intr }}$ as $0.017(\mu \Omega \mathrm{m})^{-1}$ for $\mathrm{Cu}, 0.074(\mu \Omega \mathrm{m})^{-1}$ for $\mathrm{Au}$, and $0.409(\mu \Omega \mathrm{m})^{-1}$ for Pt. These results are in good agreement with other ab initio calculations, which provided 
for Au and Pt hosts the values of 0.07-0.08 [25,26] and 0.44 [16] $(\mu \Omega \mathrm{m})^{-1}$, respectively. This shows that the Fermi-sea contribution neglected in the used Kubo-Středa formula (see the Appendix) does not exceed $8 \%$ of the total intrinsic contribution.

With the intrinsic contribution determined, the separation according to Eq. (1) is achieved. Let us perform now the decomposition of the side-jump contribution following the idea of Eq. (2). As was mentioned above, the skew scattering is solely caused by the vertex corrections. By contrast, the side-jump mechanism is not only provided by them but has also a contribution independent of the vertex corrections. This is transparent within the semiclassical approach, where the side-jump contribution to the spin current density can be written, in analogy to the AHE [5], as

$$
j_{x}^{z \mathrm{sj}}=\sigma_{x y}^{z \mathrm{sj}} E_{y}=-\frac{|e|}{V} \sum_{k} g_{k} S_{k}^{z} v_{k, x}^{\mathrm{sj}} .
$$

Here, $\mathbf{S}_{k}$ and $\mathbf{v}_{k}^{\mathrm{sj}}$ are the spin polarization [13] and the so-called side-jump velocity [20], respectively. According to Ref. [20], $\mathbf{v}_{k}^{\mathrm{sj}}$ is determined by both the scattering at impurities and the topological properties of the host crystal related to its Berry curvature [23,27]. The nonequilibrium part of the distribution function $g_{k}$ is proportional to the mean free path [28,29]

$$
\boldsymbol{\Lambda}_{k}=\boldsymbol{\Lambda}_{k}^{\text {out }}+\boldsymbol{\Lambda}_{k}^{\text {in }}
$$

consisting of the scattering-out and scattering-in terms, where the latter one corresponds to the vertex corrections of the Kubo theory [30]. Thus, Eqs. (5) and (6) support the decomposition of Eq. (2) with $\sigma_{x y}^{z \mathrm{sj}(\mathrm{nvc})}$ and $\sigma_{x y}^{z \mathrm{sj}(\mathrm{vc})}$ describing the parts independent of the vertex corrections and solely caused by them, respectively. Our procedure allows us to obtain these contributions to the side-jump mechanism separately.

Indeed, the part $\sigma_{x y}^{z \mathrm{sj}(\mathrm{vc})}$ was already derived in Ref. [10] via a procedure similar to Eq. (4) but applied to the difference of the SHCs obtained with and without vertex corrections. Here, using the same approach together with our knowledge of the total side-jump contribution, we can obtain its first part as $\sigma_{x y}^{z \mathrm{sj}(\mathrm{nvc})}=\sigma_{x y}^{z \mathrm{sj}}-\sigma_{x y}^{z \mathrm{sj}(\mathrm{vc})}$. Figure 4 shows the two parts of the side-jump contribution to the SHC separately. The most important point is that they are of comparable size. This means it is impossible to neglect one of them, but one has to consider the entire side-jump contribution to the SHC. Another interesting point is related to the magnitude of the side-jump contribution comparing $\mathrm{Au}$ and $\mathrm{Pt}$ as a host. While for $\mathrm{Pt}$ the intrinsic contribution $\sigma_{x y}^{z i n t r}$ is about six times larger than for $\mathrm{Au}$, there is no similar enhancement for $\sigma_{x y}^{z \mathrm{sj}}$. In other words, the influence of the Berry curvature on the side-jump mechanism is not as important as the scattering properties caused by impurities.

This finding supports the main assumption of the resonant scattering model proposed by Fert and Levy [12,18] for the estimation of the side-jump contribution to the SHE. Indeed, their approach does not include the influence of the host band structure via the Berry curvature, due to the restriction to systems for which the spherical band approximation can be justified. Taking into account that the vertex corrections are also neglected in the Fert-Levy model, one could assume
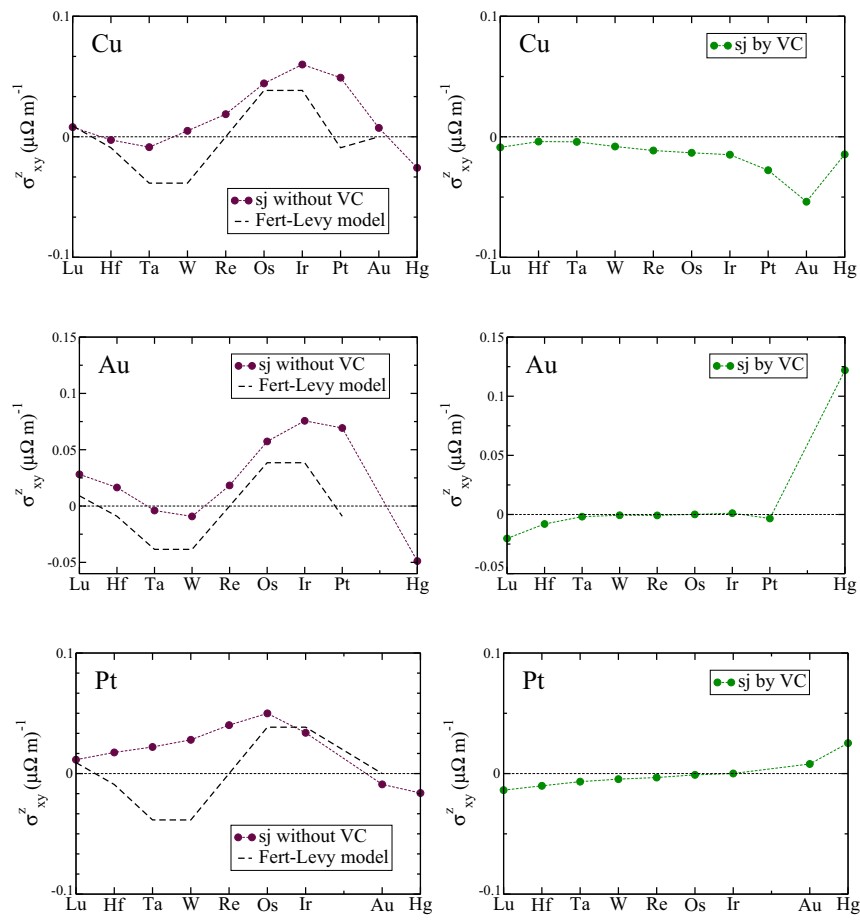

FIG. 4. (Color online) The two parts of the side-jump contribution, $\sigma_{x y}^{z \mathrm{sj}(\mathrm{nvc})}$ (left) and $\sigma_{x y}^{z \mathrm{sj}(\mathrm{vc})}$ (right), are shown for different dilute alloys based on $\mathrm{Cu}, \mathrm{Au}$, and $\mathrm{Pt}$ hosts. The dashed curves in the left panels are plotted (in arbitrary units) according to Eq. (7) obtained within the Fert-Levy model [12].

that it may describe $\sigma_{x y}^{z \mathrm{sj}(\mathrm{nvc})}$ for hosts with a free-electron-like Fermi surface. For the considered systems, the scattering phase shift of $p$ electron states in the expression derived for this contribution in Ref. [12] has no significant influence on its structure. Therefore, we neglect it and obtain

$$
\sigma_{x y}^{z \mathrm{si}(\mathrm{nvc})} \sim\left(1-4 \sin ^{2} \eta_{2}\right) \sin 2 \eta_{2} .
$$

Here, $\eta_{2}$ is the scattering phase shift corresponding to the impurity $d$ states. Within the resonant scattering model this quantity can be approximated by $\frac{\pi Z_{d}}{10}$ [12], where $Z_{d}$ is the number of impurity $d$ electrons which changes from 1 for $\mathrm{Lu}$ to 10 for $\mathrm{Au}$ impurities. As shown by Fig. 4, for $\mathrm{Cu}$ and $\mathrm{Au}$ hosts such a model estimation provides reasonable qualitative agreement with the calculated $\sigma_{x y}^{z \mathrm{sj}(\mathrm{nvc})}$. This is not the case for platinum because of its complex Fermi surface [31]. The model-based results also strongly disagree with $\sigma_{x y}^{z \mathrm{sj}(\mathrm{vc})}$. This needs to be kept in mind for comparison of the side-jump contribution obtained within the two different approximations mentioned in the Introduction.

It is important to mention that our results clarify the situation concerning a long-standing question about the magnitude of the side-jump contribution [3,4,7,32,33]. While it is commonly believed that in the dilute limit the skew-scattering mechanism should be dominating [9-12], there was no clear understanding whether the side-jump contribution may ever be significant as well. Various estimations aimed to elucidate this point but based on simple general arguments led to different conclusions. For instance, Crépieux and Bruno stated in Ref. [32] that it is impossible to predict which of the two 
extrinsic mechanisms should dominate in the high-disorder regime, while Sushkov et al. [33] concluded that the sidejump contribution is generally negligible. Based on our firstprinciples calculations, we show that the two contributions can be comparable even at impurity concentrations of a few at. \%. The same conclusion was obtained by Fert and Levy based on their impurity-specific model consideration [12]. This demonstrates that an adequate description of the electron scattering at impurities is essential, in order to derive a reasonable estimation of the side-jump contribution.

Finally, it is desirable to have a scheme to determine $\sigma_{x y}^{z i n t r}$ by considering dilute alloys instead of ideal crystals. In contrast to the latter ones, the aforementioned scheme based on the broadening via a complex energy is not necessary to ensure the convergence using a reasonable number of $\mathbf{k}$ points [24]. This idea can be realized in the following way. As was discussed above, applying the procedure of Eq. (4) to the total SHC and the difference between the total SHC and its counterpart calculated without the vertex corrections, we obtain $\sigma_{x y}^{z \text { intr }}+\sigma_{x y}^{z \mathrm{sj}}$ and $\sigma_{x y}^{z \mathrm{sj}(\mathrm{vc})}$, respectively. This allows us to separate the resulting sum $\sigma_{x y}^{z \text { intr }}+\sigma_{x y}^{z \mathrm{sj}(\mathrm{nvc})}$ as $\sigma_{x y}^{z \text { intr }}+\sigma_{x y}^{z \mathrm{sj}}-\sigma_{x y}^{z \mathrm{sj}(\mathrm{vc})}$. Now let us take into account that generally the side-jump contribution depends on the type of impurity atoms solved in the host. By contrast, the intrinsic contribution, entirely provided by the band structure of the related ideal crystal, is impurity independent. Consequently, by an appropriate choice of impurities it should be possible to obtain the case $\left|\sigma_{x y}^{z \text { intr }}\right| \gg\left|\sigma_{x y}^{z \mathrm{sj}(\mathrm{nvc})}\right|$, that would provide a good estimation for the intrinsic contribution as the dominant one in the known sum $\sigma_{x y}^{z i n t r}+\sigma_{x y}^{z s j(n v c)}$. Impurities, which fulfill the required condition, could be recognized by a statistical analysis of results obtained for a large number of different alloys based on the same host. However, one can reduce the computational effort by reasonable predictions of impurities possessing negligible side-jump mechanism. One class of possible candidates is related to light atoms with $s$ character of valence electron states. Indeed, the SOC induced by them should be weak because of both the small atomic number and the vanishing atomic orbital moment. From this perspective, $\mathrm{Li}, \mathrm{Be}, \mathrm{Na}$, and $\mathrm{Mg}$ impurities can be taken, in order to estimate the intrinsic contribution.

Following this route, we have performed additional calculations considering the four light impurities in $\mathrm{Cu}, \mathrm{Au}$, and Pt hosts. The sum $\sigma_{x y}^{z \text { intr }}+\sigma_{x y}^{z \mathrm{sj}(\mathrm{nvc})}$ obtained according to the procedure explained above is shown in Fig. 5. Evidently, the chosen impurities provide a good estimate for the intrinsic contribution. By averaging over the four considered alloys for each host, we evaluate $\sigma_{x y}^{\text {zintr }}$ as $0.016(\mu \Omega \mathrm{m})^{-1}$ for $\mathrm{Cu}, 0.088$ $(\mu \Omega \mathrm{m})^{-1}$ for $\mathrm{Au}$, and $0.404(\mu \Omega \mathrm{m})^{-1}$ for Pt. These values are in good agreement with those obtained by the calculations discussed above, which are shown as horizontal dashed lines in Fig. 5 for comparison. Thus, this way to estimate the intrinsic contribution can be used as a cheaper alternative to the direct but computationally demanding calculations.

\section{SUMMARY}

We propose an accurate procedure for the separation of the intrinsic, side-jump, and skew-scattering contributions to the

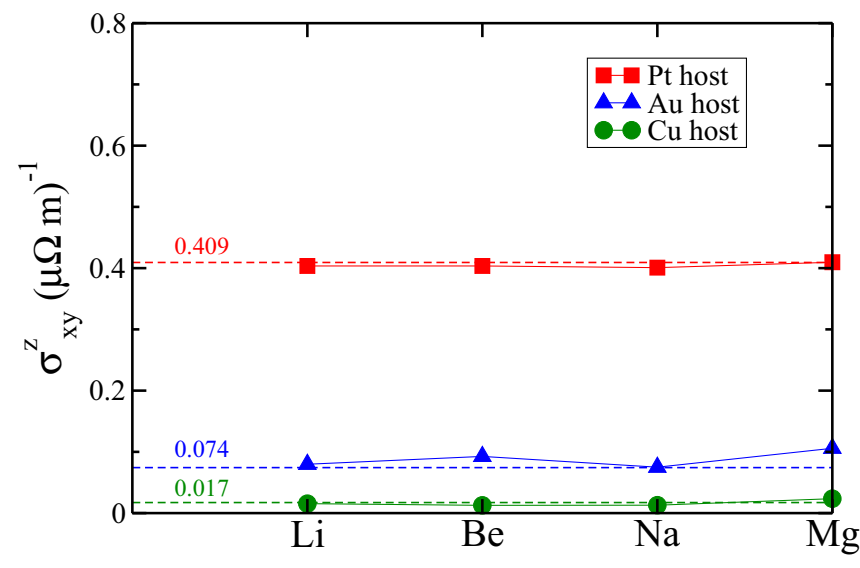

FIG. 5. (Color online) The sum of the intrinsic contribution $\sigma_{x y}^{z i n t r}$ and the first part of the side-jump contribution $\sigma_{x y}^{z \mathrm{sj}(\mathrm{nvc})}$ calculated for $\mathrm{Li}, \mathrm{Be}, \mathrm{Na}$, and $\mathrm{Mg}$ impurities in $\mathrm{Cu}$ (green circles), Au (blue triangles), and $\mathrm{Pt}$ (red squares) hosts is shown in comparison to the corresponding intrinsic contribution (dashed lines and numbers) obtained from its straightforward calculations.

spin Hall conductivity within one and the same computational method based on the first-principles Kubo-Středa approach. This is applied to various dilute alloys based on $\mathrm{Cu}, \mathrm{Au}$, and Pt hosts, which especially clarifies the influence of the vertex corrections on the side-jump mechanism. The presented scheme opens a way for further deeper theoretical investigations of the spin Hall effect with a possible elucidation of its dominating mechanisms.

\section{ACKNOWLEDGMENTS}

The Munich and Halle groups acknowledge support by the Deutsche Forschungsgemeinschaft (DFG) via SFB 689 and SFB 762, respectively. M.G. acknowledges financial support from the DFG via a research fellowship (GR3838/1-1). D.V.F. would like to thank N. A. Sinitsyn for enlightening discussions on the side-jump mechanism.

\section{APPENDIX: COMPUTATIONAL DETAILS}

Based on the Kubo-Středa linear-response theory [32,34], the presented spin Hall conductivity was calculated as a correlation function describing the connection between a spin-current $\hat{\mathbf{J}}$ induced by a charge current $\hat{\mathbf{j}}$ :

$$
\begin{aligned}
\sigma_{\mathrm{xy}}^{\mathrm{z}}= & \frac{\hbar}{4 \pi N \Omega} \operatorname{Tr}\left(\hat{J}_{\mathrm{x}}^{\mathrm{z}}\left(\hat{G}^{+}-\hat{G}^{-}\right) \hat{j}_{\mathrm{y}} \hat{G}^{-}\right. \\
& \left.-\hat{J}_{\mathrm{x}}^{\mathrm{z}} \hat{G}^{+} \hat{j}_{\mathrm{y}}\left(\hat{G}^{+}-\hat{G}^{-}\right)\right\rangle_{c},
\end{aligned}
$$

where $\Omega$ is the unit-cell volume and $N$ refers to the number of sites. This expression involves the $y$ component of the relativistic current density operator $\hat{j}_{\mathrm{y}}=-|e| c \alpha_{y}$ and the $z$ component of the relativistic spin-polarization current density operator $[24,35,36]$ with the current density along the $x$ direction: $\hat{J}_{\mathrm{x}}^{z}=|e| c \alpha_{\mathrm{x}}\left(\beta \Sigma_{\mathrm{z}}-\frac{\gamma_{5} \hat{p}_{\mathrm{z}}}{m c}\right)$. Here, $\alpha$, $\beta$, and $\gamma_{5}$ are the standard Dirac matrices and $\Sigma_{z}$ refers to the $z$ component of the vector of the relativistic spin 
matrices $(\mu=x, y, z)[37,38]$ :

$$
\begin{aligned}
& \alpha_{\mu}=\left(\begin{array}{ll}
0_{2} & \sigma_{\mu} \\
\sigma_{\mu} & 0_{2}
\end{array}\right), \quad \beta=\left(\begin{array}{cc}
\mathbb{1}_{2} & 0_{2} \\
0_{2} & -\mathbb{1}_{2}
\end{array}\right), \\
& \gamma_{5}=\left(\begin{array}{cc}
0_{2} & -\mathbb{1}_{2} \\
-\mathbb{1}_{2} & 0_{2}
\end{array}\right), \quad \Sigma_{z}=\left(\begin{array}{ll}
\sigma_{z} & 0_{2} \\
0_{2} & \sigma_{z}
\end{array}\right)
\end{aligned}
$$

with $\sigma_{\mu}$ being the Pauli matrices.

Equation (A1) was used for the pure host crystals as well as the alloys considered in our work. To describe the effect of random substitutional disorder for the latter ones, we use the coherent potential approximation (CPA) [30,39]. For this case the brackets $\langle\ldots\rangle_{c}$ in Eq. (A1) indicate a configurational average treated by means of CPA. The calculation of vertex corrections appearing as a difference between correlated and uncorrelated configurational averages $\left\langle\hat{J}_{\mathrm{x}}^{\mathrm{z}} \hat{G}^{+} \hat{j}_{\mathrm{y}} \hat{G}^{-}\right\rangle_{c}$ and $\left\langle\hat{J}_{\mathrm{x}}^{\mathrm{z}} \hat{G}^{+}\right\rangle_{c}\left\langle\hat{j}_{\mathrm{y}} \hat{G}^{-}\right\rangle_{c}$, respectively, is based on the CPA transport formalism introduced by Butler [30]. Note that the latter slightly differs by the one introduced by Velický [39] and used, e.g., in the TB-LMTO approach [40].

Further note that in Eq. (A1) a term related to the orbital current has been neglected as it was done previously $[10,40]$. For cubic crystals, which is the case for the systems considered in the presented work, this term has been shown to be small [41]. In the case of the anomalous Hall conductivity, the neglected term is equivalent to the Fermi-sea term [40] present in the Bastin equation [42], which represents a sum over all occupied states and cannot affect a description of skew scattering or side jump but the intrinsic mechanism. It was demonstrated that for cubic systems the Fermi-sea contribution is significantly smaller in comparison to the dominant Fermisurface contribution [41]. Here, we can estimate the amount of the Fermi-sea contribution by comparing our results obtained for the pure host crystals with those from Refs. [16,25,26] based on the Berry curvature calculations including both contributions. This allows us to conclude that the missing contribution does not exceed $8 \%$ of the complete intrinsic contribution to the SHC.

For our first-principles calculations, we either take into account the vertex corrections in Eq. (A1) or completely skip them from the consideration, obtaining either the total SHC $\sigma_{x y}^{z}$ or its part called $\sigma_{x y}^{z(\mathrm{nvc})}$, respectively. Their difference gives us the contribution $\sigma_{x y}^{z(\mathrm{vc})}$ which is solely caused by the vertex corrections. The obtained decomposition

$$
\sigma_{x y}^{z}=\sigma_{x y}^{z(\mathrm{nvc})}+\sigma_{x y}^{z(\mathrm{vc})}
$$

is extended further as

$$
\sigma_{x y}^{z(\mathrm{nvc})}=\sigma_{x y}^{z \text { intr }}+\sigma_{x y}^{z \mathrm{j}(\mathrm{nvc})}
$$

and

$$
\sigma_{x y}^{z(\mathrm{vc})}=\sigma_{x y}^{z \text { skew }}+\sigma_{x y}^{z \mathrm{sj}(\mathrm{vc})} .
$$

This procedure is schematically represented by Fig. 1 and explained in detail above. The crucial point is the linear behavior of $\sigma_{x y}^{z}$ or $\sigma_{x y}^{z(\mathrm{vc})}$ as a function of the longitudinal charge conductivity in the dilute limit, where both $\sigma_{x y}^{z \text { skew }}$ and $\sigma_{x x}$ are inversely proportional to the impurity concentration. Depending on the magnitude of the intrinsic and side-jump contributions, the well-pronounced linear behavior illustrated by Fig. 2 can be obtained at different concentrations for different alloys. A significant decrease of the impurity concentration would require an enormous increase of the number of $\mathbf{k}$ points in the Brillouin zone. However, for the considered systems the necessary linear dependence of the SHC as a function of $\sigma_{x x}$ was achieved with impurity concentrations above 0.5 at. \%, which allowed us to use the reasonable number of $\mathbf{k}$ points mentioned below.

The $G^{+}$and $G^{-}$present in Eq. (A1) are the retarded and advanced Green functions evaluated at the Fermi level by means of the relativistic Korringa-Kohn-Rostoker method [43] and obtained as $G^{ \pm}\left(\mathbf{r}, \mathbf{r}^{\prime}, E\right)=\lim _{\eta \rightarrow 0^{+}} G\left(\mathbf{r}, \mathbf{r}^{\prime}, E \pm i \eta\right)$. The Green function in the real-space multiple-scattering representation is given by (see Ref. [44], and references therein)

$$
\begin{aligned}
G\left(\mathbf{r}, \mathbf{r}^{\prime}, E_{F}\right)= & \sum_{\Lambda \Lambda^{\prime}} Z_{\Lambda}^{n}\left(\mathbf{r}, E_{F}\right) \tau_{\Lambda \Lambda^{\prime}}^{n m}\left(E_{F}\right) Z_{\Lambda^{\prime}}^{m \times}\left(\mathbf{r}^{\prime}, E_{F}\right) \\
& -\delta_{n m} \sum_{\Lambda}\left[Z_{\Lambda}^{n}\left(\mathbf{r}, E_{F}\right) J_{\Lambda}^{n \times}\left(\mathbf{r}^{\prime}, E_{F}\right) \Theta\left(r_{n}^{\prime}-r_{n}\right)\right. \\
& \left.+J_{\Lambda}^{n}\left(\mathbf{r}, E_{F}\right) Z_{\Lambda}^{n \times}\left(\mathbf{r}^{\prime}, E_{F}\right) \Theta\left(r_{n}-r_{n}^{\prime}\right)\right]
\end{aligned}
$$

with the four-component site-centered wave functions $Z_{\Lambda}^{n}\left(\mathbf{r}, E_{F}\right)$ and $J_{\Lambda}^{n}\left(\mathbf{r}, E_{F}\right)$ being regular and irregular solutions to the single-site Dirac equation. Here, $\Lambda=(\kappa, \mu)$ represents the relativistic quantum numbers and $\tau$ is the scattering path operator.

The calculations were performed using the atomic sphere approximation for the potential. A wave-function expansion with angular momentum cutoff $l_{\max }=3$ was used. For the investigated alloys, impurity concentrations in the range $0.5-5$ at. $\%$ were considered with the corresponding number of $\mathbf{k}$ points in the $\mathrm{BZ}$ as $10^{9}$ to $10^{7}$ to ensure convergence. In case of the pure crystals, a small imaginary part $\epsilon$ between $10^{-6}$ and $10^{-4}$ Ry was added to the Fermi energy with using about $10^{9} \mathbf{k}$ points.
[1] M. I. Dyakonov and V. Perel, Phys. Lett. A 35, 459 (1971); J. E. Hirsch, Phys. Rev. Lett. 83, 1834 (1999).

[2] R. Karplus and J. M. Luttinger, Phys. Rev. 95, 1154 (1954); E. Adams and E. Blount, J. Phys. Chem. Solids 10, 286 (1959).

[3] J. Smit, Physica 21, 877 (1955); 24, 39 (1958).

[4] L. Berger, Physica 30, 1141 (1964); Phys. Rev. B 2, 4559 (1970); 5, 1862 (1972).
[5] N. A. Sinitsyn, J. Phys.: Condens. Matter 20, 023201 (2008).

[6] N. Nagaosa, J. Sinova, S. Onoda, A. H. MacDonald, and N. P. Ong, Rev. Mod. Phys. 82, 1539 (2010).

[7] J. Sinova, S. O. Valenzuela, J. Wunderlich, C. H. Back, and T. Jungwirth, arXiv:1411.3249.

[8] J. Sinova, D. Culcer, Q. Niu, N. A. Sinitsyn, T. Jungwirth, and A. H. MacDonald, Phys. Rev. Lett. 92, 126603 (2004). 
[9] S. Onoda, N. Sugimoto, and N. Nagaosa, Phys. Rev. Lett. 97, 126602 (2006).

[10] S. Lowitzer, M. Gradhand, D. Ködderitzsch, D. V. Fedorov, I. Mertig, and H. Ebert, Phys. Rev. Lett. 106, 056601 (2011).

[11] Y. Niimi, M. Morota, D. H. Wei, C. Deranlot, M. Basletic, A. Hamzic, A. Fert, and Y. Otani, Phys. Rev. Lett. 106, 126601 (2011).

[12] A. Fert and P. M. Levy, Phys. Rev. Lett. 106, 157208 (2011).

[13] M. Gradhand, D. V. Fedorov, P. Zahn, and I. Mertig, Phys. Rev. Lett. 104, 186403 (2010); Phys. Rev. B 81, 245109 (2010).

[14] Z. Fang, N. Nagaosa, K. S. Takahashi, A. Asamitsu, R. Mathieu, T. Ogasawara, H. Yamada, M. Kawasaki, Y. Tokura, and K. Terakura, Science 302, 92 (2003).

[15] G. Y. Guo, Y. Yao, and Q. Niu, Phys. Rev. Lett. 94, 226601 (2005).

[16] G. Y. Guo, S. Murakami, T.-W. Chen, and N. Nagaosa, Phys. Rev. Lett. 100, 096401 (2008).

[17] J. Weischenberg, F. Freimuth, J. Sinova, S. Blügel, and Y. Mokrousov, Phys. Rev. Lett. 107, 106601 (2011).

[18] P. M. Levy, Phys. Rev. B 38, 6779 (1988).

[19] In Ref. [10] the remaining part of the spin Hall conductivity was simply called intrinsic contribution, although it is influenced by the side-jump mechanism described without the vertex corrections. Therefore, this one should be distinguished from the conventional intrinsic contribution, represented by $\sigma_{x y}^{z \text { intr }}$ in Eq. (1), being a pure feature of the host band structure.

[20] N. A. Sinitsyn, Q. Niu, and A. H. MacDonald, Phys. Rev. B 73, 075318 (2006).

[21] N. A. Sinitsyn, A. H. MacDonald, T. Jungwirth, V. K. Dugaev, and J. Sinova, Phys. Rev. B 75, 045315 (2007).

[22] Throughout the paper we use for the SHC the same units and sign as in Ref. [10]. This means the values of $\sigma_{x y}^{z}$ presented here correspond to the ones of $\sigma_{y x}^{s}$ considered in Ref. [29]. Their relation to other possible conventions used in literature was exhaustively discussed in Ref. [45].

[23] M. V. Berry, Proc. R. Soc. London A 392, 45 (1984).

[24] S. Lowitzer, D. Ködderitzsch, and H. Ebert, Phys. Rev. Lett. 105, 266604 (2010).
[25] Y. Yao and Z. Fang, Phys. Rev. Lett. 95, 156601 (2005).

[26] G. Y. Guo, J. Appl. Phys. 105, 07C701 (2009).

[27] M. Gradhand, D. V. Fedorov, F. Pientka, P. Zahn, I. Mertig, and B. L. Györffy, J. Phys.: Condens. Matter 24, 213202 (2012).

[28] I. Mertig, Rep. Prog. Phys. 62, 237 (1999).

[29] C. Herschbach, D. V. Fedorov, I. Mertig, M. Gradhand, K. Chadova, H. Ebert, and D. Ködderitzsch, Phys. Rev. B 88, 205102 (2013).

[30] W. H. Butler, Phys. Rev. B 31, 3260 (1985).

[31] A. Johansson, C. Herschbach, D. V. Fedorov, M. Gradhand, and I. Mertig, J. Phys.: Condens. Matter 26, 274207 (2014).

[32] A. Crépieux and P. Bruno, Phys. Rev. B 64, 014416 (2001).

[33] O. P. Sushkov, A. I. Milstein, M. Mori, and S. Maekawa, Europhys. Lett. 103, 47003 (2013).

[34] P. Středa, J. Phys. C 15, L717 (1982).

[35] A. Vernes, B. L. Györffy, and P. Weinberger, Phys. Rev. B 76, 012408 (2007)

[36] S. Lowitzer, D. Ködderitzsch, and H. Ebert, Phys. Rev. B 82, 140402(R) (2010).

[37] M. E. Rose, Elementary Theory of Angular Momentum (Wiley, New York, 1957).

[38] M. E. Rose, Relativistic Electron Theory (Wiley, New York, 1961).

[39] B. Velický, Phys. Rev. 184, 614 (1969).

[40] I. Turek, J. Kudrnovský, and V. Drchal, Phys. Rev. B 86, 014405 (2012).

[41] T. Naito, D. S. Hirashima, and H. Kontani, Phys. Rev. B 81, 195111 (2010)

[42] A. Bastin, C. Lewiner, O. Betbeder-Matibet, and P. Nozieres, J. Phys. Chem. Solids 32, 1811 (1971).

[43] H. Ebert, in Electronic Structure and Physical Properties of Solids, Lecture Notes in Physics Vol. 535, edited by H. Dreyssé (Springer, Berlin, 2000), p. 191.

[44] H. Ebert, D. Ködderitzsch, and J. Minár, Rep. Prog. Phys. 74, 096501 (2011).

[45] D. V. Fedorov, C. Herschbach, A. Johansson, S. Ostanin, I. Mertig, M. Gradhand, K. Chadova, D. Ködderitzsch, and H. Ebert, Phys. Rev. B 88, 085116 (2013). 